\title{
Evaluating NeuroSENSE for assessing depth of hypnosis during desflurane anesthesia: an adaptive, randomized-controlled trial
}

\section{Une évaluation du moniteur NeuroSENSE pour mesurer la profondeur de l'hypnose pendant une anesthésie au desflurane : une étude randomisée contrôlée adaptable}

\author{
Matthias Görges, PhD (1) - Nicholas C. West, MSc (i) • Erin M. Cooke, BSc (1) • \\ Shanshan Pi, MStat (i) - Rollin F. Brant, PhD (i) - Guy A. Dumont, PhD (1) • \\ J. Mark Ansermino, MBBCh, MSc, FRCPC (1) • Richard N. Merchant, MD, FRCPC
}

Received: 18 March 2019/Revised: 12 August 2019/Accepted: 28 October 2019/Published online: 5 November 2019

(C) Canadian Anesthesiologists' Society 2019

\begin{abstract}
Purpose Processed electroencephalography (EEG) monitors support depth-of-hypnosis assessment during anesthesia. This randomized-controlled trial investigated the performance of the NeuroSENSE electroencephalography (EEG) monitor to determine whether its wavelet anesthetic value for central nervous system $\left(W A V_{C N S}\right)$ index distinguishes consciousness from unconsciousness during induction of anesthesia (as assessed by the anesthesiologist) and emergence from anesthesia (indicated by patient responsiveness), and whether it correlates with changes in desflurane
\end{abstract}

M. Görges, PhD $(\bowtie) \cdot$ E. M. Cooke, BSc .

J. M. Ansermino, MBBCh, MSc, FRCPC

Department of Anesthesiology, Pharmacology \& Therapeutics,

University of British Columbia, Vancouver, BC, Canada

e-mail: mgorges@bcchr.ca

BC Children's Hospital Research Institute, 950 West 28th

Avenue, Vancouver, BC V5Z 4H4, Canada

N. C. West, MSc

Department of Anesthesiology, Pharmacology \& Therapeutics,

University of British Columbia, Vancouver, BC, Canada

S. Pi, MStat

Department of Statistics, University of British Columbia,

Vancouver, BC, Canada

R. F. Brant, $\mathrm{PhD}$

BC Children's Hospital Research Institute, 950 West 28th

Avenue, Vancouver, BC V5Z 4H4, Canada minimum alveolar concentration (MAC) during maintenance of anesthesia.

Methods EEG was collected using a fronto-temporal bilateral montage. The WAV $V_{C N S}$ was continuously recorded by the NeuroSENSE monitor, to which the anesthesiologist was blinded. Anesthesia was induced with propofol/remifentanil and maintained with desflurane, with randomized changes of $-0.4,0$, or +0.4 $M A C$ every 7.5 min within the 0.8-1.6 MAC range, if clinically acceptable to the anesthesiologist. During emergence from anesthesia, desflurane was stepped down by 0.2 MAC every five minutes.

Department of Statistics, University of British Columbia, Vancouver, BC, Canada

G. A. Dumont, $\mathrm{PhD}$

BC Children's Hospital Research Institute, 950 West 28th Avenue, Vancouver, BC V5Z 4H4, Canada

Department of Electrical Engineering, University of British Columbia, Vancouver, BC, Canada

R. N. Merchant, MD, FRCPC

Department of Anesthesiology, Pharmacology \& Therapeutics, University of British Columbia, Vancouver, BC, Canada

Department of Anesthesia, Royal Columbian Hospital, Fraser Health Authority, New Westminster, BC, Canada 
Results Data from 75 patients with a median [interquartile range] age of 41[35-52] yr were obtained. The WAV $V_{C N S}$ distinguished consciousness from unconsciousness as assessed by the anesthesiologist, with area under the receiver operating characteristic curve of 99.5\% (95\% confidence interval [CI], 98.5 to 100.0) at loss of consciousness and 99.4\% (95\% CI, 98.5 to 100.0) at return of consciousness. Bilateral WAV $V_{C N S}$ changes correlated with desflurane concentrations, with -8.0 and $-8.6 W A V_{C N S}$ units, respectively, per 1 MAC change in the 0.8-1.6 MAC range during maintenance of anesthesia and -10.0 and $-10.5 W A V_{C N S}$ units, respectively, in the 0.4 1.6 MAC range including emergence from anesthesia.

Conclusion The NeuroSENSE monitor can reliably discriminate between consciousness and unconsciousness, as assessed by the anesthesiologist, during induction of anesthesia and with a lower level of reliability during emergence from anesthesia. The $W A V_{C N S}$ correlates with desflurane concentration but plateaus at higher concentrations, similar to other EEG monitors, which suggests limited utility to titrate higher concentrations of anesthetic vapour.

Trial registration clinicaltrials.gov, NCT02088671; registered 17 March, 2014.

\section{Résumé}

Objectif Cette étude randomisée contrôlée a évalué la performance du moniteur d'électroencéphalographie (EEG) NeuroSENSE afin de déterminer si sa valeur anesthésique d'ondelette pour l'indice $d u$ système nerveux central (wavelet anesthetic value for central nervous system, WA $\left.V_{S N C}\right)$ était capable de distinguer la conscience de l'inconscience pendant l'induction de l'anesthésie (telle qu'évaluée par l'anesthésiologiste) et le réveil de l'anesthésie (tel qu'indiqué par la réactivité du patient), et si cette valeur était corrélée aux modifications de la concentration alvéolaire minimale (MAC) de desflurane pendant le maintien de l'anesthésie.

Méthode Les données électro-encéphalographiques ont été récoltées à l'aide d'un monitorage fronto-temporal bilatéral. La WAV $V_{S N C}$ a été enregistrée en continu par le moniteur NeuroSENSE, dont les données étaient tenues cachées de l'anesthésiologiste. L'anesthésie a été induite à l'aide de propofol / rémifentanil et maintenue avec du desflurane, avec des modifications randomisées de -0,4, 0 ou +0,4 MAC toutes les 7,5 min dans une plage de MAC de 0,8-1,6. Pendant le réveil de l'anesthésie, le desflurane a été réduit de 0,2 MAC toutes les cinq minutes.

Résultats Les données de 75 patients d'un âge médian [écart interquartile] de 41 [35-52] ans ont été obtenues. La $W A V_{S N C}$ a différencié la conscience de l'inconscience avec une surface sous la courbe de fonction d'efficacité de l'observateur de 99,5\% (intervalle de confiance [IC] 95
$\%, 98,5$ à 100,0) au moment de la perte de conscience et de 99,4\% (IC $95 \%, 98,5$ à 100,0) au rétablissement de la conscience. Les modifications bilatérales de la WAV $V_{S N C}$ étaient corrélées aux concentrations de desflurane, avec des unités de $W A V_{S N C}$ de $-8,0$ et $-8,6$, respectivement par unité de MAC dans la plage de MAC de 0,8-1,6 pendant le maintien de l'anesthésie et de -10,0 et -10,5 unités $W A V_{S N C}$, respectivement, dans la plage de MAC de 0,41,6, incluant le réveil de l'anesthésie.

Conclusion Le moniteur NeuroSENSE peut distinguer de façon fiable un état de conscience d'un état d'inconscience pendant l'induction de l'anesthésie et, à un degré de fiabilité moindre, pendant le réveil de l'anesthésie. La $W A V_{S N C}$ est corrélée à la concentration de desflurane mais atteint un plateau à des concentrations plus élevées, tout comme les autres moniteurs EEG, ce qui suggère une utilité limitée pour titrer de plus fortes concentrations de gaz anesthésiques.

Enregistrement de l'étude clinicaltrials.gov, NCT02088671; enregistrée le 17 mars 2014.

Anesthesiologists assess depth of hypnosis (DOH) by integrating physiologic variables (e.g., blood pressure, heart rate) with clinical signs (e.g., sweating, tearing, movement) $^{1}$; for example, loss of verbal response and eyelash reflex as well as hemodynamic and muscle tone changes determine whether the patient is ready for laryngoscopy. ${ }^{2,3}$ Processed electroencephalography (EEG) monitors provide a continuous, objective measure of the effects of hypnotic agents for $\mathrm{DOH}$ assessment during anesthesia. $^{3-7}$ These monitors promise reduced incidence of intraoperative awareness, reduced drug consumption, and faster recovery. ${ }^{8,9}$ While they may improve outcomes, ${ }^{10}$ their clinical benefit is still debated. ${ }^{6,8}$

The NeuroSENSE monitor (NeuroWave Systems Inc., Cleveland, OH, USA; see Fig. 1) reports the wavelet anesthetic value for central nervous system monitoring $\left(\mathrm{WAV}_{\mathrm{CNS}}\right)$, a $0-100 \mathrm{DOH}$ index, ${ }^{11}$ with $40-60$ representing the target range for general anesthesia. ${ }^{12,13}$ This monitor uses wavelet analysis (a mathematical timeand-frequency transformation) of the normalized EEG signal in the gamma frequency band. In this approach, each one-second epoch of EEG data is analyzed and the "wavelet coefficients calculated from the EEG are pooled into a statistical representation, which is then compared with two well-defined states: the awake state with normal EEG activity, and the isoelectric state with maximal cortical depression". The $\mathrm{WAV}_{\mathrm{CNS}}$ index quantifies the DOH based on the probability of the analyzed EEG epoch belonging to either of these two extreme states. ${ }^{11}$ The 


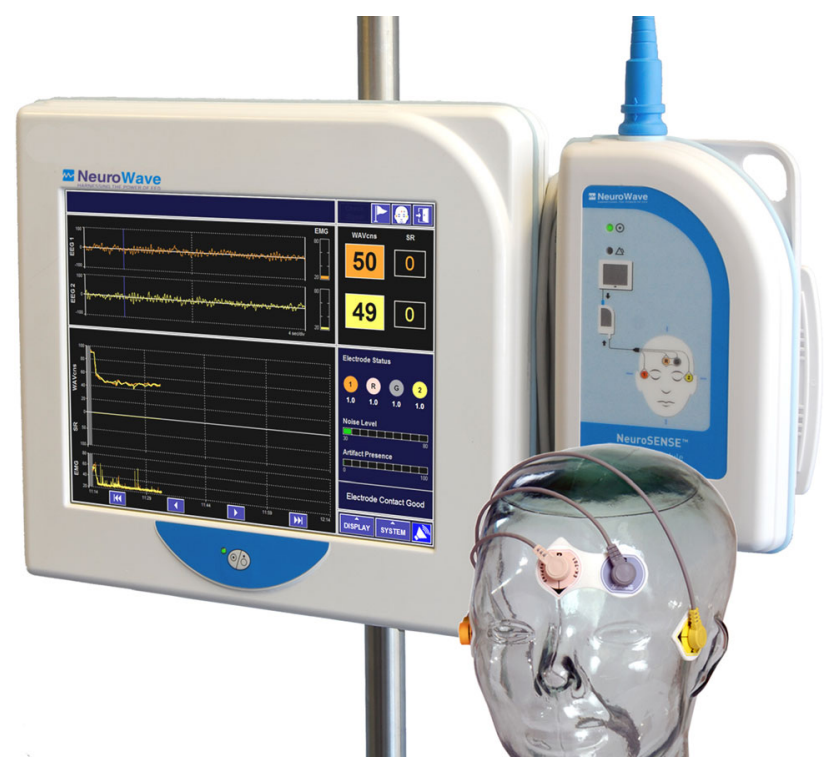

Fig. 1 NeuroSENSE NS-701 depth-of-hypnosis monitoring system. The figure shows the display module on the left, the patient module on the right, and the EasyPrep Electrode Kit EK-701 attached to a glass head to show electrode montage below. Used with permission of NeuroWave Systems Inc.

shaping function of the $\mathrm{WAV}_{\mathrm{CNS}}$ index was created to use the same range as the bispectral index (BIS) (Medtronic, Minneapolis, MN, USA). ${ }^{11}$

The NeuroSENSE monitor has been approved and is used clinically in Canada and Europe. While used clinically by anesthesiologists, there are limited data substantiating the manufacturer's claim that the $\mathrm{WAV}_{\mathrm{CNS}}$ index can be used to quantify hypnotic drug effect. It has been evaluated in a closed-loop system for propofolremifentanil anesthesia, ${ }^{14,15}$ but its performance during inhalational anesthesia has only been reported in a small $(n$ = 24) study comparing its index with the BIS. ${ }^{16}$

It is a matter of debate whether DOH indices provided by processed EEG monitors provide an objective clinical measure of the state of the patient's consciousness or depth of unconsciousness. For the purpose of this study, we consider "consciousness" to indicate an awake state (verbal responses and obeying commands) and "unconsciousness" to indicate an anesthetized state (where these responses are no longer present), as assessed by the anesthesiologist.

The aim of this study was to investigate whether the NeuroSENSE can serve as a clinically useful DOH monitor. The primary hypothesis was that $\mathrm{WAV}_{\mathrm{CNS}}$ distinguishes consciousness from unconsciousness (as assessed by the anesthesiologist) during induction of anesthesia. We then evaluated whether the $\mathrm{WAV}_{\mathrm{CNS}}$ correlated with randomized changes in desflurane concentration and accurately indicated return of consciousness (as indicated by patient responsiveness). Finally, while processed EEG monitors are not expected to predict response to stimulation, ${ }^{17}$ we aimed to evaluate the ability of the $\mathrm{WAV}_{\mathrm{CNS}}$ to predict patient response to intubation.

\section{Methods}

Study design

We conducted a step-randomized, prospective, blinded, clinical trial to evaluate the effectiveness of the NeuroSENSE NS-701 monitor (Fig. 1) for assessing DOH during the induction of anesthesia with propofol, and the maintenance and emergence stages of general anesthesia with desflurane. The anesthesiologist was blinded to the NeuroSENSE monitor throughout the study. The anesthesiologist assessed depth of anesthesia using their clinical judgement and experience, both to guide their clinical management of the patient and to accept or reject study procedures (see randomization).

The study was registered (Clinical Trials registration no. NCT02088671, first posted 17 March, 2014) and approved by Fraser Health Research Ethics Board (FHREB 2013121, first approved 12 February, 2014). All study procedures were performed in accordance with the ethical standards of this committee and the 1964 Helsinki declaration and its amendments. Written informed consent was obtained from all participants. Data are reported according to STROBE guidelines.

\section{Patient population}

The study was conducted at Eagle Ridge Hospital (Port Moody, BC, Canada) and Royal Columbian Hospital (New Westminster, BC, Canada) between 3 June, 2014 and 18 December, 2014, with study closeout delayed by the sponsor until 29 September, 2016.

Eligibility criteria included adult patients (18-75 yr), an American Society of Anesthesiologists' physical status (ASA) I-III, and scheduled for elective surgery (abdominal, breast, varicose vein stripping, or orthopedic) under general anesthesia, expected to last at least one hour.

Exclusion criteria included major head injury, scalp/ skull abnormalities, recent trauma, active neurologic disorder, possible interference with the anesthetic regime (major antipsychotic medications within seven days, history of alcohol or drug abuse within 30 days), uncontrolled hypertension, bradycardia, insulin-dependent diabetes mellitus, or pregnancy. 
Conduct of the study

EasyPrep Electrode Kit EK-701 electrodes (NeuroWave Systems Inc., Cleveland, OH, USA) were attached before induction of anesthesia as per manufacturer instructions: one EEG channel electrode was placed lateral of each eye, with the reference electrode placed medial in line with the nose, approximately 1.5 inches from the mid-eye line (Fig. 1). The ground electrode shares a pad with the reference and is placed on the forehead to the left-hand side. On initial placement of the electrode, the NeuroSENSE monitor runs an impedance check and electrode-skin impedances were kept below $10 \mathrm{k} \Omega$ to ensure optimal contact.

\section{Induction of anesthesia}

Following insertion of an intravenous cannula, a $20 \mathrm{mg}$ bolus of lidocaine was administered. Anesthesia was induced with $1.5 \mathrm{mg} \cdot \mathrm{kg}^{-1}$ propofol over $30 \mathrm{sec}$; additional boluses of $0.5 \mathrm{mg} \cdot \mathrm{kg}^{-1}$ were permitted at the anesthesiologist's discretion, based on clinical response. Next, a $2.0 \mu \mathrm{g} \cdot \mathrm{kg}^{-1}$ remifentanil bolus was given. $\mathrm{Bag} / \mathrm{mask}$ ventilation commenced when the patient became apneic. Laryngoscopy was performed $60 \mathrm{sec}$ after loss of consciousness as determined by the anesthesiologists using their customary clinical criteria, without neuromuscular blocking agent, as is routine practice for this anesthetic technique at both study hospitals. Once the larynx had been visualized, 1-2 $\mathrm{mg} \cdot \mathrm{kg}^{-1}$ aerosolized lidocaine was applied to the larynx and trachea. The endotracheal tube was placed and the airway secured, after which a neuromuscular blocking agent could be given if required.

\section{Maintenance of anesthesia}

Anesthesia was maintained with inhaled desflurane in oxygen/air. The initial desflurane concentration was set at an end-tidal target of 1.2 minimum alveolar concentration (MAC), equivalent to approximately $6 \%$ desflurane, using the GE/Datex age-adjusted control algorithm of the Aisys anesthesia machine (GE Healthcare, Chicago, IL, USA). Intravenous fentanyl was administered as a $0.5 \mu \mathrm{g} \cdot \mathrm{kg}^{-1}$ bolus (rounded to the nearest $25 \mu \mathrm{g}$ ) according to clinical need. Surgical start time was not protocolized, but happened after initiation of desflurane in all cases.

\section{Randomization of desflurane concentration}

After a post-intubation steady state had been reached, a change in desflurane concentration was proposed every 7.5 min by an algorithm running on the study laptop, using a biased-coin design. For every MAC level, there was a $1 / 3$ chance of remaining at the current level, and a $2 / 3$ chance of changing in steps of $\pm 0.4 \mathrm{MAC}$; levels were limited to $0.8,1.2$, and 1.6 MAC. If the anesthesiologist considered the change acceptable, the new MAC target was set in the end-tidal control module of the anesthesia machine; otherwise, it remained at the current level for another 7.5 min. If the new randomized target was the same as the current target, the anesthesiologist was informed that no change was required. Randomization could be stopped at any time if clinically indicated, and would restart at the 1.2 MAC level when the anesthesiologist deemed it appropriate, using the same algorithm.

\section{Emergence from anesthesia}

To facilitate rapid recovery, the emergence path started 20 min before the end of surgery as estimated by the anesthesiologist. The desflurane concentration was stepped down from 0.8 MAC, in steps of 0.2 MAC, approximately every five minutes. At any time, a 0.5 $\mu \mathrm{g} \cdot \mathrm{kg}^{-1}$ fentanyl bolus could be administered to maintain analgesia. A single dose of morphine $(2.5 \mathrm{mg})$ or hydromorphone $(0.5 \mathrm{mg})$ could be administered for postoperative analgesia.

\section{Data handling}

\section{NeuroSENSE data capture}

Electroencephalographic signals were continuously recorded at a sampling frequency of $900 \mathrm{~Hz}$ by the NeuroSENSE monitor (model NS-701, software version 2.0.0.3) from a fronto-temporal bilateral sensor montage using the EasyPrep Electrode Kit EK-701 as described above. The $\mathrm{WAV}_{\mathrm{CNS}}$ was calculated and recorded in real time. The default window length for the trending algorithm was used in all cases, as recommended by the device manual; it is adaptive, shortening in response to fast changes and lengthening in response to noise, but is constrained within five to $60 \mathrm{sec}$. The anesthesiologist was blinded to the NeuroSENSE monitor throughout; only the impedance, noise level, and artifact indicators were available to a research assistant to ensure good data quality. The NeuroSENSE monitor aims to detect and remove artifacts including ocular activity, epileptic spikes, and electro-surgical interference. ${ }^{13}$ If the signal quality drops below 50\%, the monitor does not report the $\mathrm{WAV}_{\mathrm{CNS}}$. If the $\mathrm{WAV}_{\mathrm{CNS}}$ from either channel was missing or undefined, it was excluded from the analysis. 


\section{Determining loss of consciousness}

At the start of the lidocaine injection (event $\mathrm{E}_{0}$ ), before the propofol induction bolus was administered, patients were considered conscious. Two events were recorded to confirm loss of consciousness: the time of dropping a weighted ball placed in the participant's hand $\left(\mathrm{E}_{1}\right)$; and the time immediately prior to airway instrumentation, i.e., insertion of the laryngoscope $\left(\mathrm{E}_{2}\right)$. For data analysis purposes, $E_{2}$ was taken as the primary indicator of loss of consciousness, as it was deemed more reliable.

\section{Determining response to stimulation (airway instrumentation)}

The anesthesiologist identified any response to laryngoscopy, lidocaine aerosol spray, or intubation such as movement, cough, change in blood pressure, change in heart rate, etc. There were no pre-specified cut-offs for a magnitude of response constituting responsiveness, but rather the anesthesiologists used their customary clinical criteria. The two-second median $\mathrm{WAV}_{\mathrm{CNS}}$ at time $\mathrm{E}_{2}$ was used for predicting response to stimulation (airway instrumentation).

\section{Determining return of consciousness}

The start of emergence $\left(\mathrm{E}_{3}\right)$ was identified, coinciding with setting the desflurane dosing to 0.8 MAC to start the stepdown process; at this point, patients were considered unconscious. Two events were recorded to identify return of consciousness: the time patients opened their eyes when stimulated with gentle shaking/prodding at least every one minute $\left(\mathrm{E}_{4}\right)$; or verbal response to a simple question $\left(\mathrm{E}_{5}\right)$ ("do you feel any pain?").

\section{Statistical analysis}

The area under the receiver operating characteristic (ROC) curve (AUC) for discriminating between consciousness and unconsciousness during propofol induction of anesthesia have been reported as $89 \%$ and $94 \%$ for BIS (Medtronic, Minneapolis, MN, USA), 98\% for NeuroSENSE, and 93\% for Narcotrend (MonitorTechnik, Bad Bramstedt, Germany). ${ }^{18,19}$ An averaged AUC value of 94\% was taken as the expected performance for a good $\mathrm{DOH}$ monitor, with a minimal clinically useful AUC of $85 \%$ as the null hypothesis value. Using a critical alpha of 0.05 and a power of $80 \%$, MedCalc (Medcalc Software, Ostend, Belgium) was used to identify that 64 participants were required to show a statistically significant difference between $94 \%$ and $85 \%$. Considering a potential dropout rate of $20 \%$, the sample size was adjusted to 76 participants.

\section{Analysis approach}

$\mathrm{R}$ version 3.3.2 (R Foundation for Statistical Computing, Vienna, Austria) was used for the analysis. Study data, including event timestamps, were transcribed into an Excel (Microsoft, Redmond, WA, USA) table, and verified by the research assistant (E.C.). European Data Format files from the NeuroSENSE were imported into an R data frame using R's edf library. Results from each of the monitor's two bilateral EEG channels were analyzed separately, except for the AUC analyses, in which they were pooled. Demographic information was summarized using median [interquartile range (IQR)] and range.

\section{Analysis of loss/return of consciousness}

Time-series plots of $\mathrm{WAV}_{\mathrm{CNS}}$ were produced for the loss and return of consciousness transitions. For all time points, a two-second median $\mathrm{WAV}_{\mathrm{CNS}}$ (including the preceding and succeeding second of event times) was used to allow for slight variations in marker timing. The performance of the $\mathrm{WAV}_{\mathrm{CNS}}$ in discriminating consciousness from unconsciousness was evaluated using two methods: a) Wilcoxon signed rank test, with 95\% nonparametric confidence intervals (CI) and pseudo-median difference obtained using the Hodges-Lehmann estimator if no ties were encountered (otherwise, using a normal approximation approach); and b) ROC analysis, using the pROC package, ${ }^{20}$ summarized with the AUC and $95 \% \mathrm{CI}$, using a bootstrapping method, ${ }^{21}$ to denote its accuracy. To identify optimum cutoff points, we used the Youden index (maximum of the sum of sensitivity and specificity). For the loss of consciousness analysis, we analyzed both $\mathrm{E}_{0}$ to $E_{1}$, and $E_{0}$ to $E_{2}$, while for return of consciousness we used $E_{3}$ to $E_{4}$, and $E_{3}$ to $E_{5}$ (see above for definitions).

\section{Correlation of $W A V_{C N S}$ with changes in desflurane concentration}

For each case, the times of target MAC changes (up, down, or staying the same) were identified. Only the final minute of each 7.5-min episode was analyzed, to ensure a steadystate concentration had been reached. We aimed to eliminate the influence of propofol on the desflurane anesthetic effect. Unconsciousness after a bolus of propofol typically lasts for about five to ten minutes ${ }^{22}$; hence, propofol boluses were identified and the following $15 \mathrm{~min}$ of data were removed (equivalent to two 7.5-min episodes).

Generalized estimating equations (GEE) are designed to analyze repeated measures data without strong assumptions 
about within-subject correlation patterns. ${ }^{23,24}$ The GEE approach, using the geepack package, was applied to estimate the effect of changes in target MAC on serial $\mathrm{WAV}_{\mathrm{CNS}}$ measurements during the maintenance of anesthesia phase (MAC 0.8-1.6). Each participant was considered as their own data cluster to provide an appropriate grouping structure for the analysis. An independence correlation structure was applied and the usual robust (sandwich) estimator method was used to obtain standard errors. The same analysis was then repeated using the data from both the maintenance and emergence phases of anesthesia (MAC 0.4-1.6).

\section{Predicting response to stimulation (airway instrumentation)}

Using a two-second median $\mathrm{WAV}_{\mathrm{CNS}}$ at $\mathrm{E}_{2}$ and the anesthesiologist's determination of response/no response, ROC analysis was used to evaluate the performance of the $\mathrm{WAV}_{\mathrm{CNS}}$ as a predictor of response to airway instrumentation.

\section{Results}

One hundred and forty-six patients were screened for eligibility, 119 were approached for consideration in the study, and 80 gave informed consent. Four patients were excluded before study procedures were initiated (one surgery cancelled, two did not require a general anesthetic, one did not require intubation), and one participant withdrew consent in the operating room.

Hence, study data were collected from 75 participants of median [IQR] age 41 [35-52] yr, weighing 78 [64-88] kg; 18/75 (24\%) participants were male; 36/75 (48\%) patients were ASA I, 35/75 (47\%) were ASA II, and 4/75 (5\%) ASA III. The majority of procedures were breast surgeries $(n=23)$, gynecologic procedures $(n=24)$, abdominal surgeries $(n=13)$, or orthopedic procedures $(n=12)$.

Consciousness $v s$ unconsciousness during induction of anesthesia

Between the Start of Lidocaine $\left(\mathrm{E}_{0}\right)$ and the Ball Drop $\left(\mathrm{E}_{1}\right)$ events during induction of anesthesia, median differences in $\mathrm{WAV}_{\mathrm{CNS}}$ were $-21.7(95 \% \mathrm{CI},-25.7$ to -16.7$)$ and $-19.8(95 \% \mathrm{CI},-24.7$ to -14.7$)$ for the two EEG channels (see also the Table 1). Between $\mathrm{E}_{0}$ and the Pre-Airway Instrumentation $\left(\mathrm{E}_{2}\right)$ events, these differences were -37.3 (95\% CI, -40.2 to -34.4 ) and -37.5 (95\% CI, -40.2 to -34.5). Within each channel, all differences were statistically significant with $P$ values $<0.001$ (Fig. 2a).

The ROC analysis produced predictive outcome models for loss of consciousness during induction of anesthesia: these had an AUC of $99.5 \%$ (95\% CI, 98.6 to 100.0) for the Pre-Airway Instrumentation endpoint (i.e., $\mathrm{E}_{0}$ vs $\mathrm{E}_{2}$; Youden cutoff, 82.8) and 89.9\% (95\% CI, 86.1 to 93.1) for the Ball Drop endpoint (i.e., $\mathrm{E}_{0}$ vs $\mathrm{E}_{1}$; Youden cutoff, 87.8).

Table 1 Median $\mathrm{WAV}_{\mathrm{CNS}}$ at marked events and desflurane concentration levels

\begin{tabular}{|c|c|c|c|c|c|}
\hline \multirow[b]{2}{*}{ Phase of anesthesia } & \multirow[b]{2}{*}{ Event/MAC level } & \multicolumn{2}{|c|}{ Right channel } & \multicolumn{2}{|c|}{ Left channel } \\
\hline & & Samples $^{\dagger}$ & $\mathrm{WAV}_{\mathrm{CNS}} *$ & Samples $^{\dagger}$ & $\mathrm{WAV}_{\mathrm{CNS}} *$ \\
\hline \multirow[t]{3}{*}{ Induction } & $\mathrm{E}_{0}$ lidocaine injection & 74 & 91.6 [90.4-93.0 (86.7-94.8)] & 74 & 91.2 [90.1-92.9 (85.9-95.3)] \\
\hline & $\mathrm{E}_{1}$ ball drop & 74 & 71.2 [53.9-89.4 (29.7-92.8)] & 74 & $73.6[57.7-89.0(32.1-92.7)]$ \\
\hline & $E_{2}$ laryngoscopy & 74 & $55.7[47.0-62.0(25.4-92.3)]$ & 74 & $56.0[46.2-60.9(24.7-89.0)]$ \\
\hline \multirow[t]{3}{*}{ Maintenance } & MAC 0.8 & 155 & $37.3[33.3-44.9(20.4-82.1)]$ & 157 & $37.2[32.5-45.4(20.8-81.6)]$ \\
\hline & MAC 1.2 & 242 & $33.7[29.5-38.4(5.9-83.8)]$ & 243 & 33.7 [29.5-38.4 (5.9-83.8)] \\
\hline & MAC 1.6 & 112 & $32.0[27.5-38.0(0.0-92.2)]$ & 111 & 31.7 [27.5-38.0 (0.0-91.4)] \\
\hline \multirow[t]{6}{*}{ Emergence } & $E_{3}$ start of emergence & 75 & $32.6[29.5-37.3(14.4-63.7)]$ & 75 & $33.5[28.4-37.9(13.9-58.1)]$ \\
\hline & MAC 0.6 & 70 & $44.7[37.4-57.1(24.2-88.4)]$ & 70 & $45.1[36.8-54.2(24.7-87.9)]$ \\
\hline & MAC 0.4 & 46 & 59.5 [48.4-79.4 (31.3-95.4)] & 46 & 56.0 [46.5-79.6 (32.7-94.7)] \\
\hline & MAC 0.2 & 15 & 76.8 [61.4-91.4 (51.8-94.2)] & 15 & 74.4 [62.7-88.2 (52.9-93.7)] \\
\hline & $\mathrm{E}_{4}$ eye opening & 71 & 89.4 [86.5-90.9 (36.5-96.2)] & 71 & 89.5 [86.2-91.7 (34.7-95.5)] \\
\hline & $\mathrm{E}_{5}$ response to verbal question & 70 & 89.8 [88.7-90.8 (36.5-95.2)] & 68 & 89.8 [88.6-91.0 (34.7-94.6)] \\
\hline
\end{tabular}

* Median [interquartile range (range)].

$\dagger$ Number of occurrences of each event/MAC level with recorded data; for each event, this occurred at most once per participant, but each maintenance MAC level may have multiple samples per participant.

$\mathrm{MAC}=$ minimum alveolar concentration; $\mathrm{WAV}_{\mathrm{CNS}}=$ wavelet anesthetic value for central nervous system . 
(a)

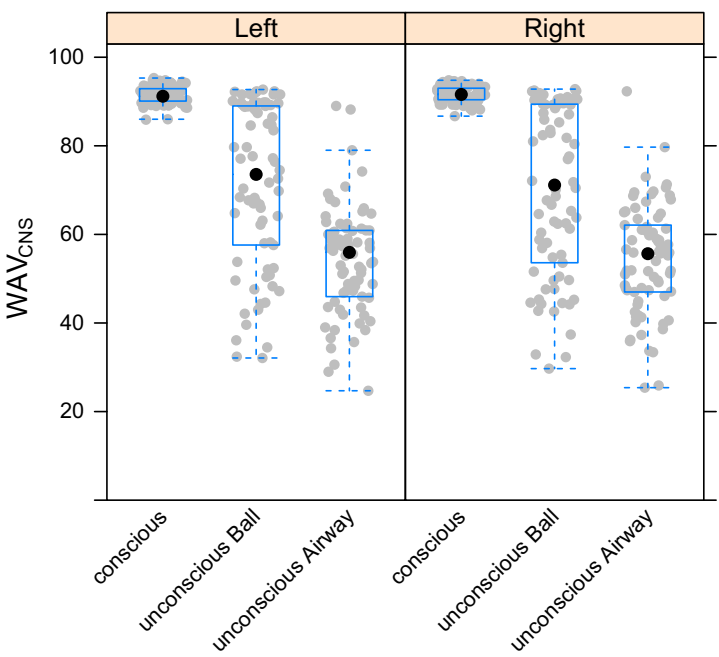

Fig. 2 Discrimination between consciousness and unconsciousness using the $\mathrm{WAV}_{\mathrm{CNS}}$ index, including: a) loss of the consciousness and b) return of consciousness. Data are presented as box and whisker plots, with the whiskers extending to the last point within $1.5 \times$ interquartile range from the edge of the box and raw data overlaid.

\section{Correlation of $\mathrm{WAV}_{\mathrm{CNS}}$ with changes in desflurane MAC}

Participants received a median [IQR] of 5 [3-6] fentanyl boluses for a cumulative dose of 200 [150-250] $\mu \mathrm{g}$ of fentanyl. During randomization of target MAC values in the maintenance period, 639 MAC randomization levels (757 including emergence) were recorded in $79.7 \mathrm{hr}$ of data. Of these, $21.0 \%$ were at $0.8 \mathrm{MAC}, 55.6 \%$ at 1.2 MAC, and $23.5 \%$ at $1.6 \mathrm{MAC}$. For each of the two EEG channels, patients spent a median [IQR] of $10.8 \%$ [0.142.6] and $11.7 \%$ [0.1-40.5] of the maintenance period in the recommended 40-60 range. The median [IQR] difference between the two EEG channels, calculated as right-left, was 0.1 [ -1.4 to 1.6$] \mathrm{WAV}_{\mathrm{CNS}}$ units $(95 \% \mathrm{CI}$, -10.8 to 7.6). These differences decreased slightly with increasing MAC: the median [IQR] differences were 1.0 [ -0.7 to 3.0$]$ at $0.2 \mathrm{MAC}, 0.6[-1.0$ to 1.9$]$ at $0.4 \mathrm{MAC}$, $0.1[-1.1$ to 1.8$]$ at $0.6 \mathrm{MAC}, 0.4[-0.9$ to 1.5$]$ at 0.8 MAC, 0.0 [ -1.4 to 1.3$]$ at $1.2 \mathrm{MAC}$, and 0.0 [ -1.7 to 1.3$]$ at $1.6 \mathrm{MAC}$.

Figure 3 shows two example case plots: the left subplot shows a case that illustrates good correlation; the right subplot shows a case illustrating poor correlation. Table 1 summarizes $\mathrm{WAV}_{\mathrm{CNS}}$ values observed at each MAC level.

The GEE model showed a normalized change in $\mathrm{WAV}_{\mathrm{CNS}}$ units for each 1 MAC change in desflurane concentration: these were -8.0 (95\% CI, -11.6 to -4.4$)$ and $-8.6(95 \% \mathrm{CI},-12.1$ to -5.1$)$ for the two EEG channels. Figure 4 shows the observed $\mathrm{WAV}_{\mathrm{CNS}}$ range for

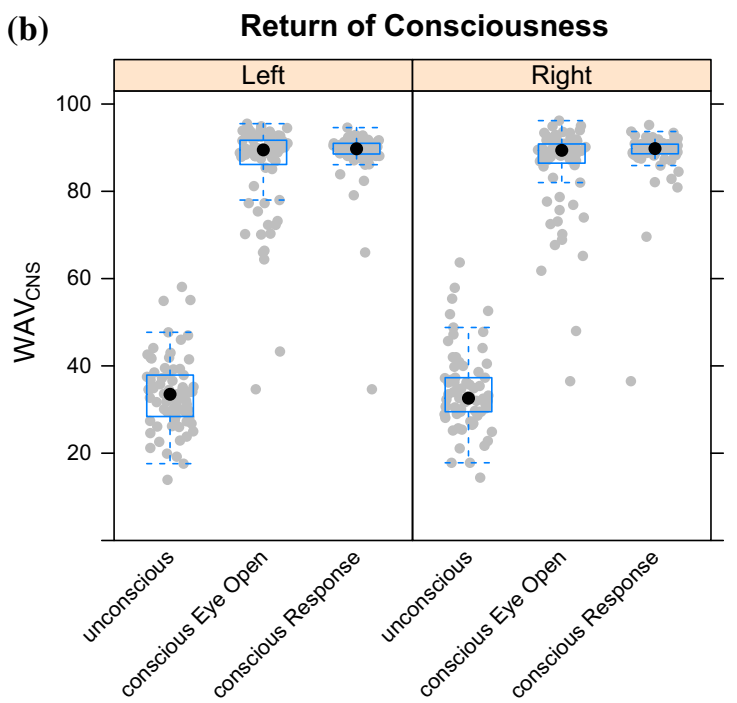

Note that for return of consciousness, there are three points observed with a $\mathrm{WAV}_{\mathrm{CNS}}<60$; all of these patients showed a much higher $\mathrm{WAV}_{\mathrm{CNS}}$ only a few seconds thereafter, suggesting a small delay in detecting very rapid increases in response to stimulation. $\mathrm{WAV}_{\mathrm{CNS}}=$ wavelet anesthetic value for central nervous system.

each MAC target; Figure 5 shows the slopes of the linear regression model, separated by the MAC target before the change.

If the emergence data are included in the GEE analysis, the sensitivity increases to -10.0 ( $95 \% \mathrm{CI},-13.4$ to -6.6$)$ and $-10.5(95 \% \mathrm{CI},-13.9$ to -7.2$) \mathrm{WAV}_{\mathrm{CNS}}$ units for the respective channels. If the data are limited to the emergence period only (target MAC $\leq 0.8$, yielding 171 observations), the sensitivities become -42.8 (95\% CI, -57.3 to -28.3$)$ and -46.6 (95\% CI, -60.8 to -32.5$)$ $\mathrm{WAV}_{\mathrm{CNS}}$ units for each channel.

Predicting response to stimulation (airway instrumentation)

During airway instrumentation, the median differences in $\mathrm{WAV}_{\mathrm{CNS}}$ between responders and non-responders were $-6.2(95 \% \mathrm{CI},-11.7$ to $-0.7 ; P=0.03)$ and $-5.6(95 \%$ CI, -10.9 to $-0.6 ; P=0.03$ ) for the two EEG channels ( $n$ $=74)$. The predictive model had an AUC of $65.3 \%(95 \%$ CI, 55.8 to 74.1) (Fig. 6).

\section{Return of consciousness}

Between the start of emergence $\left(\mathrm{E}_{3}\right)$ and eye opening $\left(\mathrm{E}_{4}\right)$ events, the median differences in $\mathrm{WAV}_{\mathrm{CNS}}$ were 52.7 (95\% CI, 49.2 to 55.8) and 52.7 (95\% CI, 49.7 to 55.6) for the two EEG channels. Between $\mathrm{E}_{3}$ and return of verbal response $\left(\mathrm{E}_{5}\right)$, these differences were $55.6(95 \% \mathrm{CI}, 52.7$ to 57.9) and 55.7 (95\% CI, 53.3 to 57.9). Again, within each 

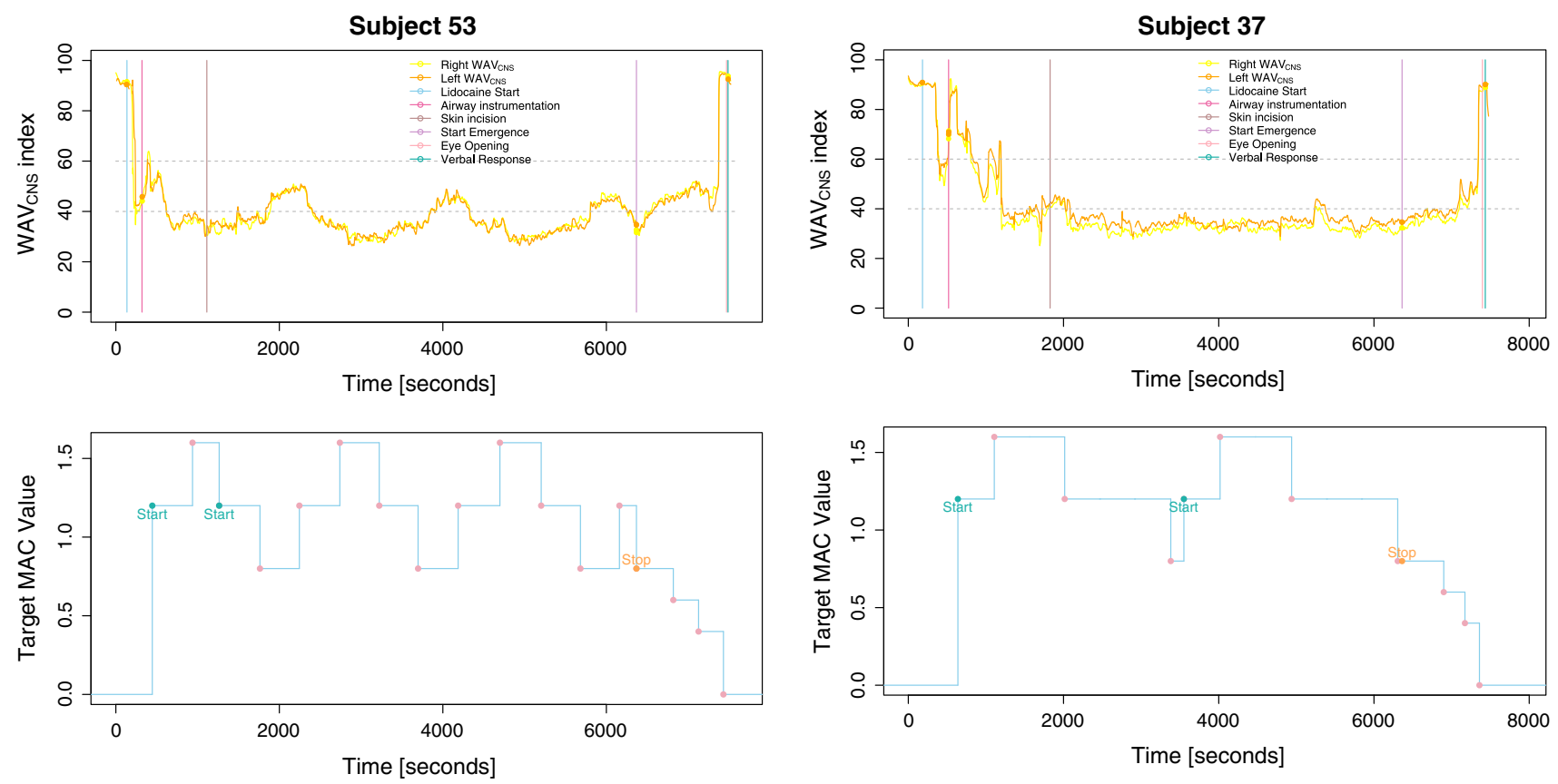

Fig. 3 Example cases with $\mathrm{WAV}_{\mathrm{CNS}}$ (top subplot) and target MAC values (bottom subplot) to demonstrate the study methodology. This case on the left shows the best correlation between changes of $\mathrm{WAV}_{\mathrm{CNS}}$ and target MAC values observed in this trial, while the case

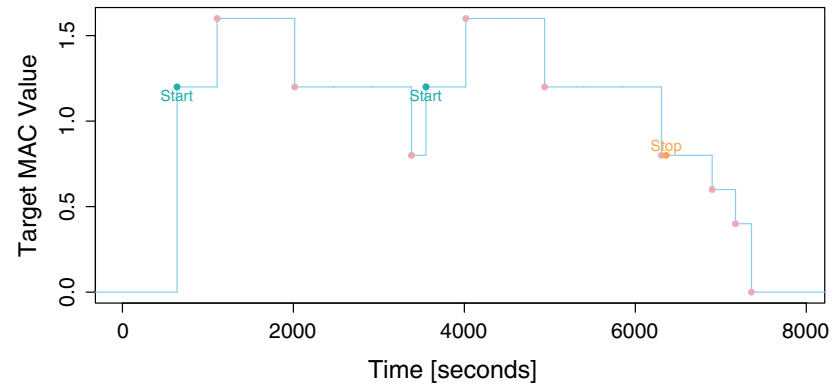

on the right shows an example with little to no correlation. MAC = minimum alveolar concentration; $\mathrm{WAV}_{\mathrm{CNS}}=$ wavelet anesthetic value for central nervous system.

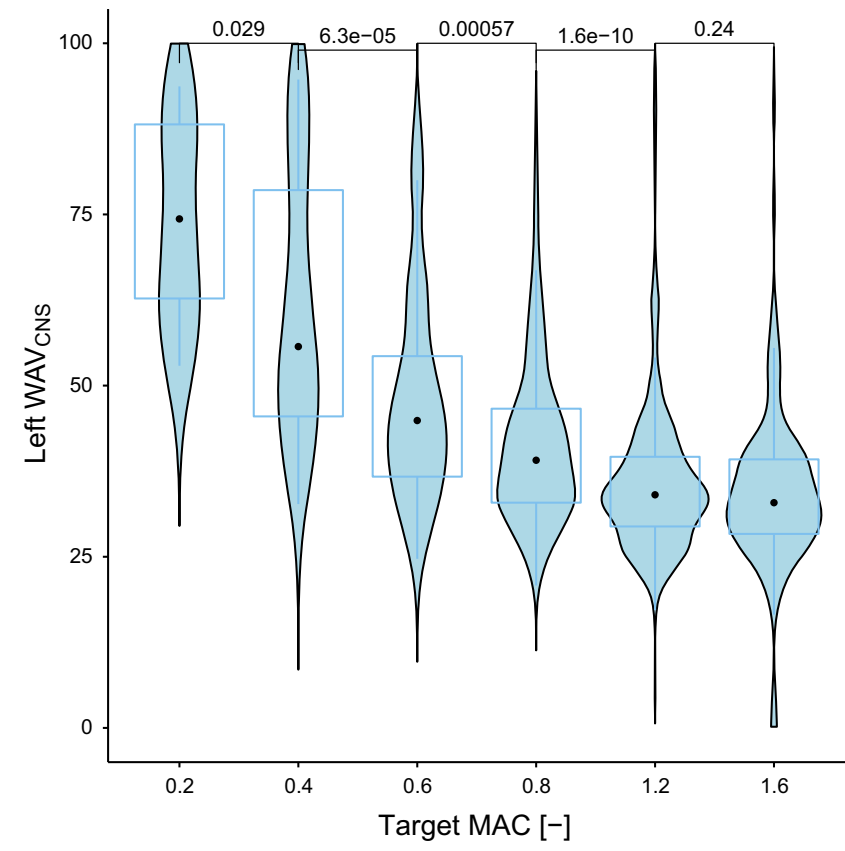

Fig. 4 Observed WAV $\mathrm{CNS}_{\mathrm{CN}}$ range for each MAC target, split by EEG channel. Data are shown as hybrid of violin plots, overlaid by boxplots, with statistical comparisons to their next neighbour indicated above; all comparisons, except for the MAC $1.2 v s \quad 1.6$

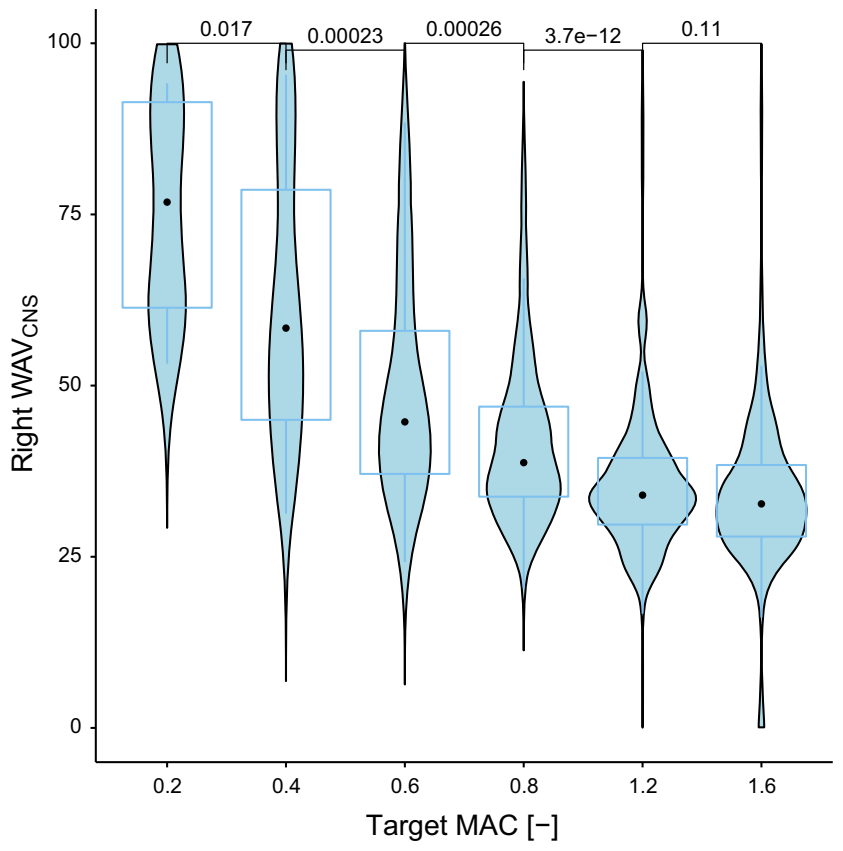

level, were statistically significant, because of the large number of samples included in the analysis. EEG = electroencephalography; $\mathrm{MAC}=$ minimum alveolar concentration; $\mathrm{WAV}_{\mathrm{CNS}}=$ wavelet anesthetic value for central nervous system. 

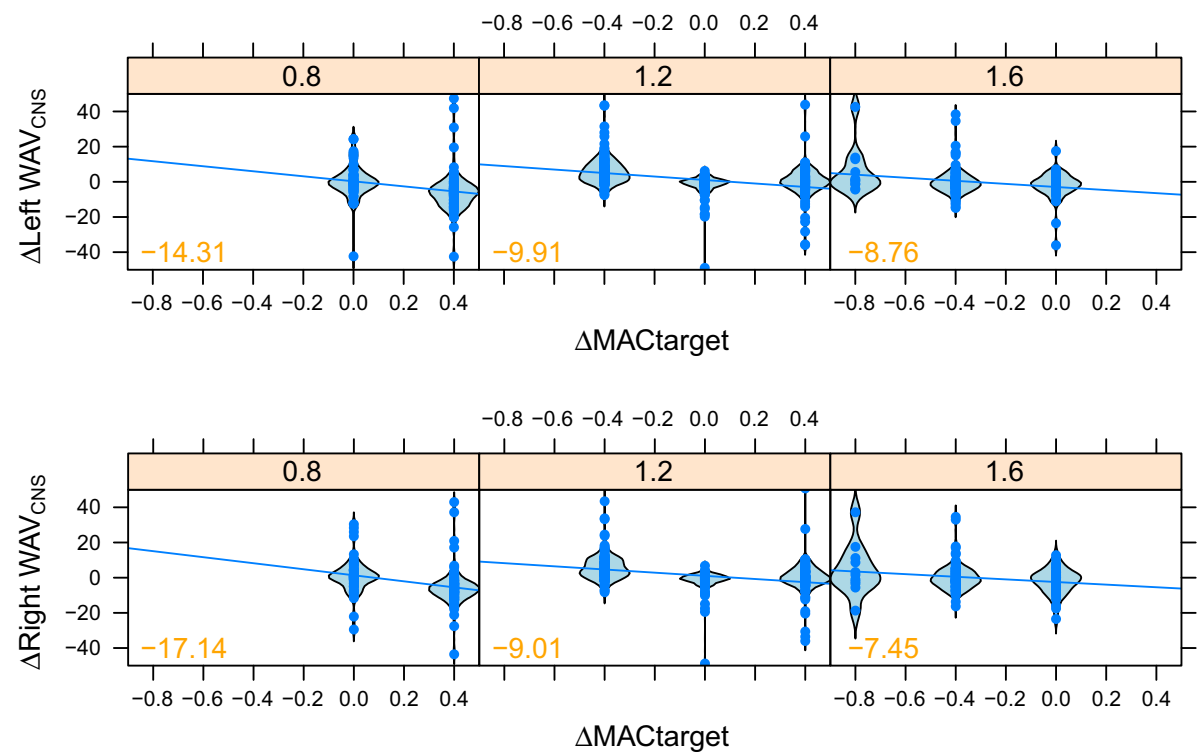

Fig. 5 Change in $\mathrm{WAV}_{\mathrm{CNS}}\left(\triangle \mathrm{WAV} \mathrm{V}_{\mathrm{CNS}}\right)$ for a given change of MAC target ( $\triangle \mathrm{MACtarget})$. The top subplot shows data from the right $\mathrm{EEG}$ channel; the bottom subplot shows data from the left EEG channel. Both subplots are split by MAC target before the change; changes to the MAC target were either $-0.4,0.0$, or $+0.4 \mathrm{MAC}$, according to the randomization schedule. Data are shown as hybrid of violin plots, overlaid with raw data as dots, as well as the linear model regression line and its slope value indicated in orange. EEG = electroencephalography; $\mathrm{MAC}=$ minimum alveolar concentration; $\mathrm{WAV}_{\mathrm{CNS}}=$ wavelet anesthetic value for central nervous system.

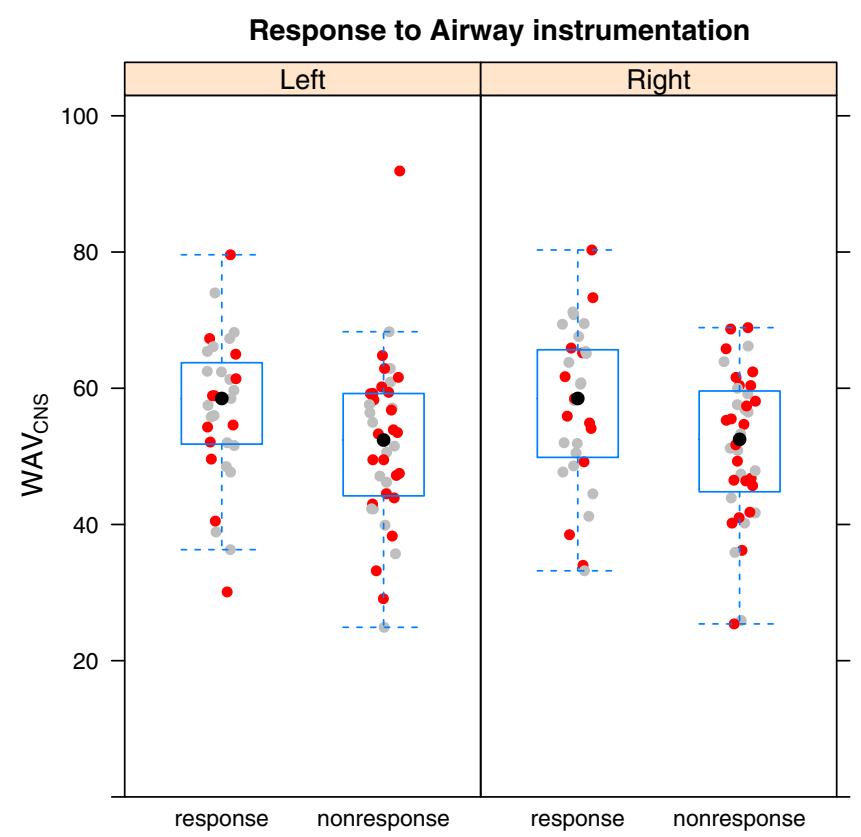

Fig. 6 Pre-stimulation $\mathrm{WAV}_{\mathrm{CNS}}$ values in responders and nonresponders to airway stimulation. In the left subplot, data are presented as box and whisker plots, with the whiskers extending to the last point within $1.5 \times$ interquartile range from the edge of the box; raw data are overlaid, with patients requiring an additional propofol bolus highlighted in red. The right subplot shows the receiver

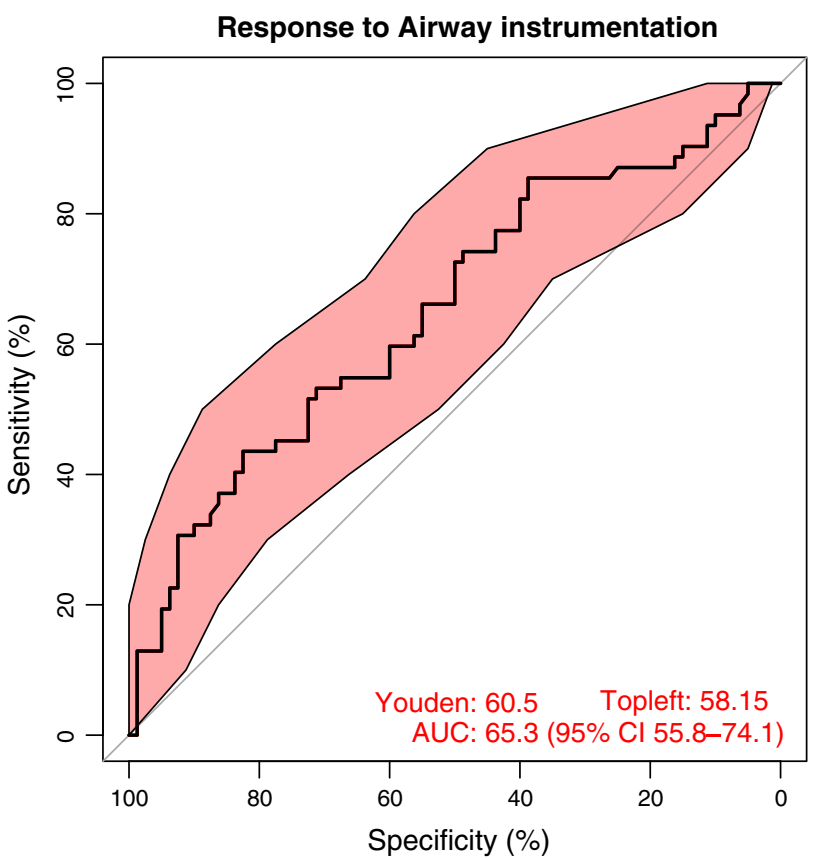

operating characteristic plot for the $\mathrm{WAV}_{\mathrm{CNS}}$ index prediction of responders, with the area under curve (AUC) and their 95\% confidence intervals for the specificity with Youden and Topleft cutoff values. $\mathrm{WAV}_{\mathrm{CNS}}=$ wavelet anesthetic value for central nervous system. 
channel, all differences were statistically significant with a $P<0.001$ (Fig. 2b).

The ROC predictive outcome models for return of consciousness had an AUC of $99.4 \%$ (95\% CI, 98.6 to 100.0 ) for the eye opening endpoint (i.e., $\mathrm{E}_{3} v s \mathrm{E}_{4}$; Youden cutoff, 60.0 ) and $99.5 \%$ (95\% CI, 98.6 to 100) for the return of verbal response endpoint (i.e., $\mathrm{E}_{3} v s \mathrm{E}_{5}$; Youden cutoff, 64.9).

\section{Discussion}

The $\mathrm{WAV}_{\mathrm{CNS}}$ was able to distinguish consciousness from unconsciousness, both during drug-induced loss of consciousness (as assessed by the anesthesiologist during propofol induction of anesthesia) and return of consciousness (as indicated by patient responsiveness during emergence from inhaled anesthesia). The predictive ROC model based on the Start of Lidocaine and Pre-Airway Instrumentation events, showed good performance, with an AUC above 99\% and a Youden cutoff value of 82.8 during induction of anesthesia; alternatively, using the Start of Lidocaine and Ball Drop events, the AUC was $90 \%$. Some overlap in $\mathrm{WAV}_{\mathrm{CNS}}$ during loss of consciousness was observed for the Ball Drop endpoint (see Fig. 2), likely a combination of the inadequacy of this endpoint (see below), and the short delay commonly seen in the physiologic response.

Correlation of $\mathrm{WAV}_{\mathrm{CNS}}$ with MAC during desflurane anesthesia

Changes in $\mathrm{WAV}_{\mathrm{CNS}}$ during maintenance of desflurane anesthesia, supplemented with fentanyl boluses as clinically indicated, correlated with changes in the ageadjusted MAC desflurane target, but this correlation may not be clinically useful. That is, the changes were small: -8.0/ -8.6 $\mathrm{WAV}_{\mathrm{CNS}}$ units per 1 MAC change in desflurane concentration in the 0.8-1.6 MAC range for each of the bilateral EEG channels. The performance improves for lower MAC values (Fig. 5). Also, with emergence data included, the sensitivity increased to $-10.0 /-10.5 \mathrm{WAV}_{\mathrm{CNS}}$ units per $1 \mathrm{MAC}$ change, for each of the two EEG channels.

The saturation effect of increasing the concentration of inhaled anesthetic seems to be independent of the EEG monitoring device used. A similar plateau effect was reported for desflurane using BIS and Narcotrend ${ }^{25}$ and for sevoflurane using BIS and Cerebral State Monitor (Danmeter $\mathrm{A} / \mathrm{S}$, Odense, Denmark). ${ }^{26}$ In a secondary analysis following a large 1,100-patient cohort study (BUnaware trial), ${ }^{27}$ Whitlock et al. found a -15.4 unit change $(95 \% \mathrm{CI},-17.8$ to -13.1$)$ for every $1 \mathrm{MAC}$ change in the $0.42-1.51$ MAC range using the BIS monitor; yet, when the authors excluded patients with extreme values, this reduced to -8.6 unit per 1 MAC change.

Different anesthetic agents have varying effects on the EEG, which affect the DOH index values calculated by EEG-based monitors. For instance, an equivalent MAC concentration of desflurane produces a lower DOH index value than sevoflurane measured by BIS and spectral edge frequency monitoring ${ }^{28}$; similarly, sevoflurane produces a lower BIS value than isoflurane. ${ }^{29}$ While the EEG patterns observed with these ether-based inhalational agents are similar to propofol under 1 MAC, they produce oscillations in the theta-band above this threshold. ${ }^{30}$ Combining anesthetic agents, as is common, adds further variation.

The performance we observed may limit the utility of the NeuroSENSE for titrating anesthetic vapour concentrations in the flat part of the anesthetic agent $v s$ DOH index slope, ${ }^{9}$ seen in the 1.2-1.6 MAC range in our study, though this problem is likely not limited to this specific monitor. Nonetheless, EEG monitoring has been shown to have benefits for reducing intraoperative desflurane dosing. ${ }^{31,32}$ Kreuer et al. found that this effect was only marginal ${ }^{33}$ and Luginbühl et al. concluded that the benefit of EEG monitoring in desflurane anesthesia lay in improved patient satisfaction rather than reduced drug consumption. ${ }^{34}$ The $\mathrm{WAV}_{\mathrm{CNS}}$ values we observed suggested a DOH that was generally deeper than the manufacturer-recommended anesthetic range of 40-60. ${ }^{12,13}$ If the NeuroSENSE had been used to maintain DOH within this range, the desflurane MAC would likely have been lower, which might have resulted in a higher $\mathrm{WAV}_{\mathrm{CNS}} /$ MAC sensitivity.

Predicting response to stimulation (airway instrumentation)

The $\mathrm{WAV}_{\mathrm{CNS}}$ ability to predict response to stimulation was poor. While a statistically significant difference was found in $\mathrm{WAV}_{\mathrm{CNS}}$ between responders and non-responders to airway instrumentation, the predictive model AUC of $63.6 \%$ (95\% CI, 54.5 to 72.7 ) was poor. This result is not surprising. Depth-of-hypnosis indices mainly capture the hypnotic component of an anesthetic, although DOH index changes have been detected in response to noxious stimuli, e.g., increased variability in the $\mathrm{BIS}^{35}$ or the difference between Response and State Entropy ${ }^{36}$ (GE Healthcare, Chicago, IL, USA). Somatic responses to noxious stimulation are mediated through the spinal cord and the autonomic nervous system, rather than the cortex. Consequently, processed EEG monitoring cannot be used to predict somatic or hemodynamic reaction to a nociceptive event, ${ }^{17}$ but only observe a change after the fact. Nevertheless, while these changes may be detectable, 
they may indicate insufficient analgesia ${ }^{37}$ and are, hence, difficult to interpret.

Study limitations

A conservative range of desflurane concentrations was used in this study, which limited our ability to determine the full range of $\mathrm{WAV}_{\mathrm{CNS}}$ changes that may be observed outside the flat part of the anesthetic-agent $v s$ DOH index slope. 9,27 Thus, the secondary analysis including the emergence steps was performed, which showed, as expected, an improvement in the sensitivity of the monitor.

We assessed loss of consciousness using two different events: Ball Drop (based on conscious effort by the patient) and Pre-Airway Instrumentation (based on clinical judgement). Both are problematic and an ideal method for identifying the moment of loss of consciousness during induction of anesthesia has not been established. ${ }^{3} \mathrm{We}$ found the Ball Drop method to be less reliable: for example, one patient commented on accidentally dropping the ball, and in two cases, the ball became trapped between the patient's hand and the bed; thus, results for this approach should be interpreted with caution.

Next, we did not pool the results of the two independent bilateral EEG channels, which may have simplified the interpretation of the results, even though they provided similar results. We did this because the monitor reports the Left and Right channels separately, never combined, to allow clinicians to easily spot any hemispheric discrepancies, e.g., due to underlying pathologies, ${ }^{38}$ or to overcome artifacts captured only in one channel.

The combined effect of propofol and desflurane following intravenous induction of anesthesia has not been completely accounted for; while we removed the 15 min of data following the propofol induction boluses, using induction with an inhalational agent may have avoided this source of uncertainty.

Finally, we evaluated response to stimulation (airway instrumentation) in patients, who had not received muscles relaxant, which may not be common practice in many institutions. The finding that the $\mathrm{WAV}_{\mathrm{CNS}}$ does not predict response to stimulation effectively is unlikely to be improved in patients who do receive muscle relaxant prior to airway instrumentation.

\section{Conclusions}

The NeuroSENSE monitor can reliably determine loss of consciousness during induction of anesthesia and return of consciousness with a slightly lower level of reliability during emergence from anesthesia. The $\mathrm{WAV}_{\mathrm{CNS}}$ correlates with desflurane dosing. Nevertheless, similar to other EEG monitoring devices, the index is more sensitive at lower MAC values and progressively reaches a plateau around 1.2 MAC, which limits its utility to titrate inhaled anesthetic at higher concentrations that will typically correspond to $\mathrm{WAV}_{\mathrm{CNS}}$ values below the manufacturer's recommended anesthetic range of 40-60. As expected, it cannot be used to predict response to stimulation.

Author contributions Matthias Görges conducted the literature review, designed the study, obtained the ethical approval to conduct the research, analyzed and interpreted the findings, and drafted the manuscript. Nicholas $C$. West collected the data and drafted the manuscript. Erin M. Cooke conducted the literature review, designed the study, obtained the ethical approval to conduct the research, and collected the data. Shanshan Pi analyzed and interpreted the findings and drafted the manuscript. Rollin F. Brant analyzed and interpreted the findings. Guy A. Dumont conducted the literature review, designed the study, and obtained the ethical approval to conduct the research. $J$. Mark Ansermino conducted the literature review, designed the study, and obtained the ethical approval to conduct the research. Richard N. Merchant conducted the literature review, designed the study, and obtained the ethical approval to conduct the research. All authors critically reviewed the manuscript.

Acknowledgements The authors wish to thank all the participating patients, anesthesiologists, the surgical and nursing teams in the operating theatre, as well as Aryannah Rollinson and Sonia Brodie for their help with the data collection.

Declaration of interests Guy A. Dumont is a co-inventor of the NeuroSENSE monitor (NeuroWave Systems Inc., Cleveland Heights, OH, USA). J. Mark Ansermino and Guy A. Dumont have consulted for NeuroWave Systems Inc. Matthias Görges, J. Mark Ansermino, Guy A. Dumont, and Richard N. Merchant are party to a licensing agreement between NeuroWave Systems Inc. and the University of British Columbia for control technology. The remaining authors have no disclosures.

Funding This study was funded by NeuroWave Systems Inc. (Cleveland Heights, OH, USA), under contract from the US Army (W81XWH-06-C-0016).

Editorial responsibility This submission was handled by Dr. Gregory L. Bryson, Deputy Editor-in-Chief, Canadian Journal of Anesthesia.

\section{References}

1. Russell IF. Midazolam-alfentanil: an anaesthetic? An investigation using the isolated forearm technique. $\mathrm{Br} \mathrm{J}$ Anaesth 1993; 70: 42-6.

2. Morgan GE Jr, Mikhail MS, Murray MJ. Clinical Anesthesiology. 4th ed. NY: McGraw-Hill Medical; 2005 .

3. Ferreira AL, Nunes $C$, Mendes $J G$, Amorim $P$. Do we have today a reliable method to detect the moment of loss of consciousness during induction of general anaesthesia? Rev Esp Anestesiol Reanim 2019; 66: 93-103.

4. Bibian S, Dumont GA, Zikov T. Dynamic behavior of BIS, Mentropy and neuroSENSE brain function monitors. J Clin Monit Comput 2011; 25: 81-7. 
5. Marchant N, Sanders R, Sleigh J, et al. How electroencephalography serves the anesthesiologist. Clin EEG Neurosci 2014; 45: 22-32.

6. Shander A, Lobel GP, Mathews DM. Brain monitoring and the depth of anesthesia: another Goldilocks dilemma. Anesth Analg 2018; 126: 705-9.

7. Fahy BG, Chau DF. The technology of processed electroencephalogram monitoring devices for assessment of depth of anesthesia. Anesth Analg 2018; 126: 111-7.

8. Musialowicz T, Lahtinen P. Current status of EEG-based depthof-consciousness monitoring during general anesthesia. Curr Anesthesiol Rep 2014; 4: 251-60.

9. Escallier KE, Nadelson MR, Zhou D, Avidan MS. Monitoring the brain: processed electroencephalogram and peri-operative outcomes. Anaesthesia 2014; 69: 899-910.

10. Zorrilla-Vaca A, Healy RJ, Wu CL, Grant MC. Relation between bispectral index measurements of anesthetic depth and postoperative mortality: a meta-analysis of observational studies. Can J Anesth 2017; 64: 597-607.

11. Zikov T, Bibian S, Dumont GA, Huzmezan M, Ries CR. Quantifying cortical activity during general anesthesia using wavelet analysis. IEEE Trans Biomed Eng 2006; 53: 617-32.

12. Agrawal G, Bibian S, Zikov T. Recommended clinical range for $\mathrm{WAV}_{\mathrm{CNS}}$ index during general anesthesia. Proceedings of the 2010 Annual Meeting of the American Society of Anesthesiologists. San Diego, CA: American Society of Anesthesiologists; 2010: A1347 (abstract).

13. Bibian $S$, Zikov T. NeuroSENSE® Monitor with $\mathrm{WAV}_{\mathrm{CNS}}$ Cortical Quantifier: a Deterministic Approach to EEG Analysis. Cleveland Heights, OH: September 2018. Available from URL: http://www.neurowavesystems.com/pdfs/NeuroSENSE_White_ Paper_September_2018.pdf (accessed September 2019).

14. West $N$, Dumont GA, van Heusden K, et al. Robust closed-loop control of induction and maintenance of propofol anesthesia in children. Paediatr Anaesth 2013; 23: 712-9.

15. West $N$, van Heusden $K$, Görges $M$, et al. Design and evaluation of a closed-loop anesthesia system with robust control and safety system. Anesth Analg 2018; 127: 883-94.

16. Bresson J, Gayat E, Agrawal G, et al. A randomized controlled trial comparison of NeuroSENSE and bispectral brain monitors during propofol-based versus sevoflurane-based general anesthesia. Anesth Analg 2015; 121: 1194-201.

17. Johansen JW. Update on bispectral index monitoring. Best Pract Res Clin Anaesthesiol 2006; 20: 81-99.

18. Zikov T, Bibian S, Ries CR, Jaromczyk BS, Voltz DM. Performance of BIS and $\mathrm{WAV}_{\mathrm{CNS}}$ to predict loss of consciousness during routine propofol induction. Proceedings of the 2006 Annual Meeting of the American Society Anesthesiologists. American Society of Anesthesiologists; 2006: A1030 (abstract).

19. Kreuer $S$, Bruhn $J$, Larsen $R$, Bialas $P$, Wilhelm $W$. Comparability of Narcotrend index and bispectral index during propofol anaesthesia. Br J Anaesth 2004; 93: 235-40.

20. Robin X, Turck N, Hainard A, et al. pROC: an open-source package for $\mathrm{R}$ and $\mathrm{S}+$ to analyze and compare ROC curves. BMC Bioinformatics 2011; https://doi.org/10.1186/1471-2105-12-77.

21. Carpenter J, Bithell J. Bootstrap confidence intervals: when, which, what? A practical guide for medical statisticians. Stat Med 2000; 19: 1141-64.

22. Carmichael FJ, Haas DA. General anesthetics. In: Kalant $\mathrm{H}$, Roschlau WH (Eds). Principles of Medical Pharmacology. Oxford University Press; 1998: 278-92.

23. Liu J, Colditz GA. Optimal design of longitudinal data analysis using generalized estimating equation models. Biom J 2017; 59 : 315-30.

24. Liang $K Y$, Zeger $S L$. Longitudinal data analysis using generalized linear models. Biometrika 1986; 73: 13-22.
25. Kreuer S, Bruhn J, Ellerkmann R, Ziegeler S, Kubulus D, Wilhelm $W$. Failure of two commercial indexes and spectral parameters to reflect the pharmacodynamic effect of desflurane on EEG. J Clin Monit Comput 2008; 22: 149-58.

26. Han DW, Linares-Perdomo OJ, Lee JS, Kim JH, Kern SE. Comparison between cerebral state index and bispectral index as measures of electroencephalographic effects of sevoflurane using combined sigmoidal E(max) model. Acta Pharmacol Sin 2011; 32: 1208-14.

27. Whitlock EL, Villafranca AJ, Lin N, et al. Relationship between bispectral index values and volatile anesthetic concentrations during the maintenance phase of anesthesia in the B-Unaware trial. Anesthesiology 2011; 115: 1209-18.

28. Kanazawa $S, \quad$ Oda $Y$, Maeda $C$, Okutani $R$. Electroencephalographic effect of age-adjusted 1 MAC desflurane and sevoflurane in young, middle-aged, and elderly patients. J Anesth 2017; 31: 744-50.

29. Gupta M, Shri I, Sakia P, Govil D. Comparison of equi-minimum alveolar concentration of sevoflurane and isoflurane on bispectral index values during both wash in and wash out phases: a prospective randomised study. Indian J Anaesth 2015; 59: 79-84.

30. Purdon PL, Sampson A, Pavone KJ, Brown EN. Clinical electroencephalography for anesthesiologists: part I: background and basic signatures. Anesthesiology 2015; 123: 937-60.

31. White PF, Ma H, Tang J, Wender RH, Sloninsky A, Kariger R. Does the use of electroencephalographic bispectral index or auditory evoked potential index monitoring facilitate recovery after desflurane anesthesia in the ambulatory setting? Anesthesiology 2004; 100: 811-7.

32. Ibrahim TH, Yousef GT, Hasan AM, Eldesuky HI. Effect of bispectral index monitoring on desflurane consumption and recovery time in morbidly obese patients undergoing laparoscopic sleeve gastrectomy. Anesth Essays Res 2013; 7 : $89-93$.

33. Kreuer S, Bruhn J, Stracke C, et al. Narcotrend or bispectral index monitoring during desflurane-remifentanil anesthesia: a comparison with a standard practice protocol. Anesth Analg 2005; 101: 427-34.

34. Luginbühl $M$, Wüthrich $S$, Petersen-Felix $S$, Zbinden $A M$, Schnider TW. Different benefit of bispectal index (BIS) in desflurane and propofol anesthesia. Acta Anaesthesiol Scand 2003; 47: 165-73.

35. Sahinovic MM, Eleveld DJ, Kalmar AF, et al. Accuracy of the composite variability index as a measure of the balance between nociception and antinociception during anesthesia. Anesth Analg 2014; 119: 288-301.

36. Aho AJ, Yli-Hankala A, Lyytikäinen LP, Jäntti V. Facial muscle activity, Response Entropy, and State Entropy indices during noxious stimuli in propofol-nitrous oxide or propofol-nitrous oxide-remifentanil anaesthesia without neuromuscular block. Br J Anaesth 2009; 102: 227-33.

37. Mathews DM, Clark L, Johansen J, Matute E, Seshagiri CV. Increases in electroencephalogram and electromyogram variability are associated with an increased incidence of intraoperative somatic response. Anesth Analg 2012; 114: 75970.

38. Brodie SM, Görges M, Ansermino JM, Dumont GA, Merchant $R N$. Closed-loop control of total intravenous anesthesia during significant intraoperative blood loss: a case report. A A Case Rep 2017; 9: 239-43.

Publisher's Note Springer Nature remains neutral with regard to jurisdictional claims in published maps and institutional affiliations. 